\title{
Cuatro líneas en el conjunto de investigaciones del DUOT
}

\section{Pablo Elinbaum}

Universidad Nacional de Rosario.

Programa de Doctorado Universitat Politècnica de Catalunya

\section{RESUMEN}

En este artículo comentamos algunas investigaciones del DUOT, en diferentes fases de avance, cuyos ejes temáticos determinan cuatro líneas específicas: el estudio de los instrumentos de planificación en Cataluña, los paisajes del turismo litoral, la dimensión transversal de las infraestructuras de la movilidad y el estudio de ciudades latinoamericanas. A partir del cruce temático de las investigaciones surgen algunas conjeturas: los instrumentos de planificación en Cataluña se mueven entre la "optimización" y la "receptividad" de sus políticas; el estudio de los paisajes del turismo abre una nueva perspectiva para su comprensión y la definición de conceptos y políticas urbanísticas; la dimensión transversal de las infraestructuras de la movilidad permite establecer un lenguaje consecuente con su escala; y la repercusión de la "circulación de ideas" y el análisis morfológico de las ciudades latinoamericanas contribuye a la reivindicación de su identidad. En resumen, estas investigaciones evidencian no sólo una aportación importante a los estudios urbanos, sino una nueva especificidad, híbrida, en las líneas de investigación en urbanismo. 


\section{ABSTRACT}

In this article we present some results of the research at different progress stages by members of the Town Planning Department. They emphasize four specific themes: the planning tools in Catalunya; the landscapes of coastal tourism; the trans-scalar dimension of mobility infrastructure and the analysis of Latin American cities. According to this cross thematic research, some hypotheses emerge: planning instruments in Catalunya move between the "optimization" and the "openness" of their policies. The study of tourism landscapes opens a new perspective to understand and define concepts and urban policies; the trans-scalar dimension of mobility infrastructure enables a consistent language with its scale; and the impact of the "ideas flows" and morphological analysis of Latin American cities contributes to claim their identity. In short, these studies show not only an important contribution to urban studies but a new hybrid specificity in the urbanism research field.

\section{INTRODUCCIÓN}

El Doctorado en Urbanismo constituye un programa de tercer ciclo y cuenta con tres grupos de investigación consolidados. En el marco del doctorado, las investigaciones en curso se apoyan en un grupo de asignaturas teóricas y se desarrollan a través de seminarios de investigación ${ }^{1}$. Actualmente el Programa se organiza según cuatro grandes líneas de investigación: Teoría e Historia de la ciudad, Análisis y proyecto urbano, Análisis y proyecto territorial y Análisis y ordenación del paisaje.

Las investigaciones que presentaré expresan la transversalidad de temas canónicos, entre las transformaciones territoriales y el paisaje; entre la historia de la ciudad y el proyecto urbano; o bien entre movilidad y los espacios

\footnotetext{
${ }^{1}$ También habría que mencionar los Seminarios Internacionales de Doctorado que constituyen una instancia para presentar las investigaciones en curso realizadas en el DUOT y asimismo una invitación abierta a otras universidades y a sus respectivos programas de doctorado para dar a conocer su producción académica. Los tres seminarios realizados han consolidado este evento anual como un magnífico lugar de intercambio académico.
} 
públicos. Mi objetivo es mostrar, a través de estos cruces temáticos que enriquecen las investigaciones, aquellos trabajos en diferentes fases de avance que más han llamado mi atención. El rasgo significativo de estas investigaciones es su convergencia en cuatro líneas temáticas específicas: el estudio de los instrumentos de planificación en Cataluña, los paisajes del turismo litoral, la dimensión transversal de las infraestructuras de la movilidad y el estudio de ciudades latinoamericanas.

\section{EL ESTUDIO DE LOS INSTRUMENTOS DE PLANIFICACIÓN EN CATALUÑA}

Asistimos a una crisis del urbanismo occidental que puede ser explicada sintéticamente a través del concepto de modernidad reflexiva (Ascher 2004). El desafío disciplinar actual radica no tan sólo en una recuperación de la legitimidad social del urbanismo (Font, 2007), sino asimismo, en la renovación de su cuerpo instrumental anacrónico (Magrinya, 2010).

Cataluña cuenta con una importante tradición en planificación urbana. El urbanismo catalán destaca, frente a experiencias más descriptivas o sectoriales, por la cultura urbanística del "proyectar". Por ello los instrumentos de planificación adquieren un protagonismo central. Esto se hace evidente en todas las escalas de intervención, desde el proyecto urbano, que tiene su correlato instrumental en el planeamiento derivado, especialmente, en los planes de reforma interior, los planes parciales y planes especiales ${ }^{2}, y$ asimismo en los planes municipales. Desde 2003 el impulso del desarrollo instrumental se ha extendido a los planes supramunicipales y territoriales.

Algunas investigaciones recientes abordan especialmente los problemas de la implementación urbanística en los cascos antiguos, en los polígonos residenciales construidos en las décadas de los sesenta y setenta, y en las

\footnotetext{
${ }^{2}$ Los planes especiales han sido estudiados en el libro "Plans molt especials" de Miquel Corominas, Joaquín Sabaté y Adolf Sotoca (2005). Barcelona: Col-legi d'Arquitectes de Catalunya, Demarcació de Barcelona: Escola Sert
} 
aglomeraciones suburbanas. En relación con las políticas públicas se aborda la especificidad de los instrumentos desde su eficiencia y operatividad o desde la representatividad de las opciones de transformación urbana o territorial.

Los objetivos de las investigaciones sobre los instrumentos de planeamiento en Cataluña se mueven entre la "optimización" y la "receptividad" de sus políticas. Algunas apuntan a definir pautas para evaluar la eficiencia de los instrumentos y determinar cuáles han sido las "buenas prácticas" con respecto a los objetivos; otras a comprender de qué manera se incluyen las "demandas endógenas" en la mecánica del planeamiento.

El primer tipo de aproximación resulta evidente en el trabajo de Ana Pagliuso ${ }^{3}$ que parte de la degradación socio-ambiental de ciertos barrios, iniciada en los años 60, e intenta verificar cómo los proyectos de intervención integral se convierten en el pilar de la política de renovación urbana. Analiza las estrategias y metodologías de intervención en tres tipos de barrios y crea un sistema de indicadores para evaluar su impacto. Esta metodología le permite confirmar una de las hipótesis del trabajo: la existencia de un "ámbito óptimo" para el desarrollo de este tipo de proyectos.

Como ejemplo del segundo tipo de aproximación podríamos referirnos a la investigación de Pablo Juarez ${ }^{4}$. Aborda el estudio de los distintos procesos de intervención urbanística en tres enclaves urbanos (Trinitat Nova, el sector Parc Central del Poblenou y la plaza Lesseps), para comprender de qué manera se incorporan los rasgos locales en los instrumentos asociados a la reforma de los barrios. El autor parte del estudio del concepto de "lugar comunicativo". A partir del mismo, se pregunta de qué modo se pueden introducir los estudios de "legibilidad" en los procesos de reforma, es decir, cómo garantizar el "sentido del lugar" a través del planeamiento.

\footnotetext{
${ }^{3}$ La Llei de barris: un pas endavant en la regeneració urbana. Investigación en curso de Ana Pagliuso. Director: Joaquín Sabaté
} 


\section{LOS PAISAJES DEL TURISMO LITORAL}

Siguiendo la línea de investigación iniciada en los años noventa por Rosa Barba y por Joaquín Sabaté a través del Plan Insular de Ordenación Territorial de Tenerife (PIOT), y recientemente por algunos trabajos académicos de Joan Busquets, cabría mencionar tres investigaciones que abordan el estudio del desarrollo turístico litoral. Los tres se remontan a la década de los cincuenta y principios de los sesenta para explicar como el turismo constituye una de las actividades económicas más dinámicas en España -actualmente representa más del $10 \%$ de las exportaciones de bienes y servicios, con más de 53 millones de visitantes extranjeros. Ante la consolidación de una tradición turística, las etapas de crisis económica habrían derivado en la oportunidad para revisar y reconducir el espacio turístico después de un periodo en el que se ha puesto en riesgo la identidad de muchos territorios. El desafío actual radica en entender las lógicas que motivan los desarrollos (espontáneos y organizados) para encontrar sinergias entre las actividades y el paisaje como oportunidad, reflexionando sobre la relación entre la nueva tipología de ordenación y su estructura de soporte. Las hipótesis más osadas de estas investigaciones apuntan a definir hasta qué punto el "nuevo turismo" sería capaz de poner en valor el paisaje. No obstante, el rasgo más innovador de este grupo de investigaciones radica en las categorías de análisis e intervención que, en sí mismas, suponen una clara aportación disciplinar.

El estudio del desarrollo turístico desde una perspectiva territorial nos permite reflexionar sobre su principal recurso, el paisaje, y las distintas formas de intervenir en él. Los paisajes del turismo, a la luz de las nuevas investigaciones abren una nueva perspectiva para la comprensión de un fenómeno, pero también para la definición de nuevos conceptos urbanísticos. A través del reconocimiento morfológico de los tejidos turísticos en Mallorca, la clasificación comprehensiva de los tipos de asentamientos de ocio en Alicante y Murcia, y el

\footnotetext{
4 La construcción comunicativa del lugar: Participación ciudadana y fomento del sentido en el planeamiento contemporáneo. Investigación en curso de Pablo Juarez. Director: Joaquim Sabaté
}

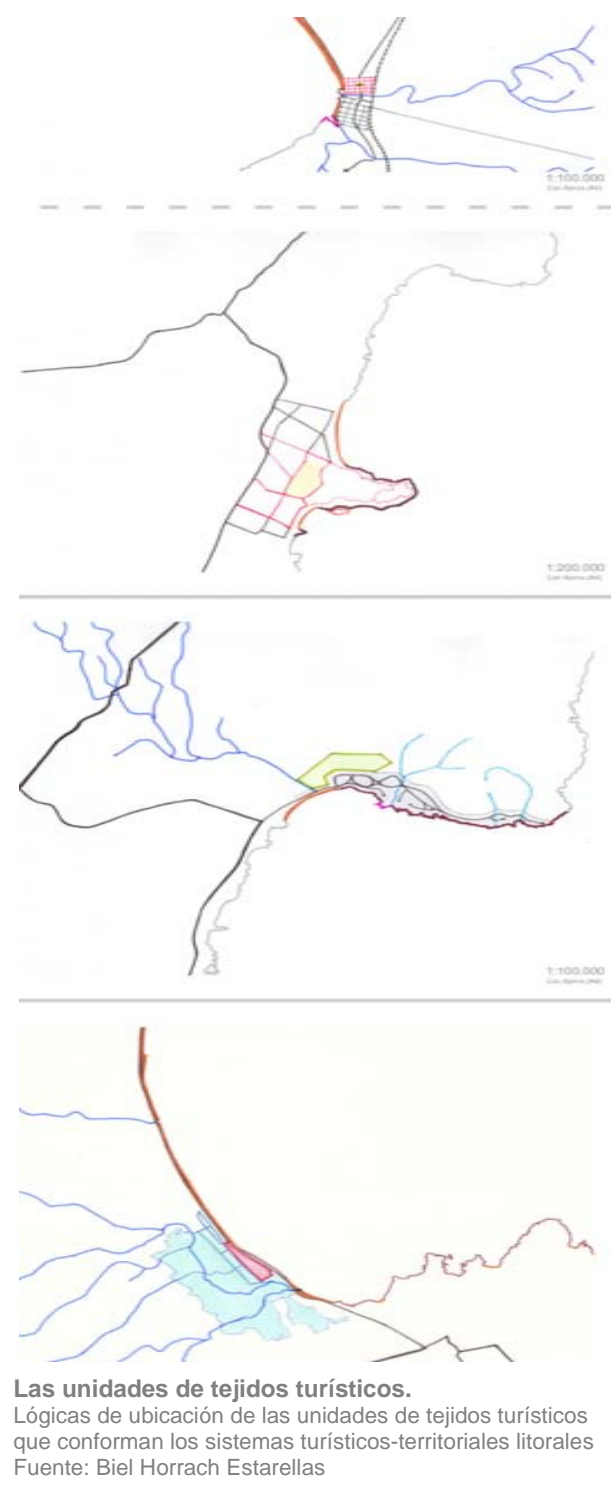


estudio del "know how" de los agentes locales en la reproducción del modelo de "sol y playa" en Lanzarote, sería posible definir tres nuevos conceptos para la comprensión del desarrollo del turismo litoral.

En un primer trabajo, Biel Horrach ${ }^{5}$ define el concepto de "sistemas turísticoterritoriales" ${ }^{6}$ y lo argumenta en dos aproximaciones. Por un lado parte de una decodificación de las razones histórico-formativas del litoral turístico de Mallorca, explicado los procesos territoriales, así como de las dinámicas socioeconómicas. En segunda instancia, parte de una lectura comprehensiva de la morfología de los tejidos turísticos, para determinar las razones históricoformativas de los nuevos tipos de conurbaciones.

En una segunda investigación, desarrollada por Pepe $\mathrm{Bru}^{7}$, se precisa el concepto de "tejido turístico-residencial". Esta tipología surge del análisis de un tramo del litoral entre los Cabos de la Nao y el de Palos, donde la maduración de los núcleos y la diversidad de los crecimientos, favorecidos por la experimentación tipológica de las células de viviendas, ha permitido, con el aumento de la densidad y el aprovechamiento de la accesibilidad existente, la sustitución paulatina de los asentamientos turísticos por residencia permanente.

Finalmente, cabría mencionar la investigación de Antonio Zamora ${ }^{8}$. Apoyado en la definición de "proyecto implícito", el autor analiza las estrategias, espontáneas e institucionales, del "modelo turístico local" confirmado posteriormente por el planeamiento. A partir de este objetivo indaga sobre las líneas maestras del "proyecto" -los argumentos paisajísticos, la imagen y la

\footnotetext{
${ }^{5}$ La Balearización. Mallorca el laboratorio de experimentación del turismo y su manifestación en el litoral. Investigación en curso de Gabriel Horrach. Director: Joaquín Sabaté

6 Previamente, el autor analiza el concepto de "unidades de tejido turístico" determinado por: los asentamientos suburbanos vacacionales, las extensiones en malla, la ciudad jardín turística y los centros autónomos.

${ }^{7}$ La ciudad de la playa. Investigación en curso de Pepe Bru. Director: Joaquín Sabaté

${ }_{8}$ Claves para una propuesta territorial. Investigación en curso de Antonio Zamora. Director: Joaquín Sabaté

${ }_{9}$ Zamora se refiere al libro de Giuseppe Dematteis (1995) "Progetto implicito.ll contributo della geografia umana alle scienze del territorio". Milano: Ed.Tipomonza
} 
identidad-, las circunstancias de su desarrollo por el gobierno insular, y pone en evidencia aquellos contenidos que se incorporarían al planeamiento vigente.

\section{LA DIMENSIÓN TRANSVERSAL DE LAS INFRAESTRUCTURAS}

Al igual que hace la tesis doctoral de Lorena Vecslir (2005) las infraestructuras de la movilidad no pueden analizarse como un sistema autónomo, esquemático y especializado. Éstas juegan un papel clave en los procesos de desarrollo de la ciudad y el territorio. Las recientes investigaciones desarrolladas en esta misma línea, desde una visión transescalar de las infraestructuras, evidencian las repercusiones en el desarrollo de nuevas ciudades, en la disyuntiva de los sistemas y los espacios públicos metropolitanos $y$, finalmente, en la arquitectura de la ciudad que determina los trazados e instalaciones de servicio. ¿En qué cuestiones radica la potencialidad del proyecto de los sistemas? ¿Qué tipo de proyecto es capaz de vincular las infraestructuras como espacio público?

Las investigaciones más ortodoxas sobre el carácter urbanístico de los sistemas restringen su estudio a los espacios canónicos como las plazas, los lugares de eventos o los lugares del ocio y el turismo. En este sentido, Barcelona constituye un caso de estudio paradigmático. ${ }^{10}$ En contraste, las investigaciones analizadas, pese a las diferentes escalas de los objetos de estudio y sin desatender la dimensión funcional, reconceptualizan la noción de espacio publico e infraestructuras. El rasgo más relevante de estas investigaciones radica en que, frente a la idea estática y fija de los elementos de la estructura urbana de la ciudad tradicional, su enfoque se centra, más allá de las escalas abordadas, en la potencialidad de los espacios marginales como espacios expectantes.

\footnotetext{
${ }^{10}$ Ver la tesis doctoral de Miquel Martí: A la recerca de les civitas contemporània. Cap a una cultura urbana de l'espai públic: L'experiència de Barcelona (1979-2003). Tesis doctoral. Director: Joaquín Sabaté. UPC, DUOT, 2004.
} 
Valga como por ejemplo en el uso del subsuelo en el contexto de la ciudad compacta occidental o en el "sistema crítico"11 asociado a los grandes corredores metropolitanos.

La manifestación explícita y autónoma de las infraestructuras, el principal capital fijo de la ciudad y el territorio, justifica el interés y la pertinencia del proyecto del espacio de "lo público", capaz de establecer un lenguaje consecuente con su escala. Destacamos dos investigaciones que mantienen un enfoque común en la forma que abordan la problemática urbanística de los sistemas y la conceptualización del espacio público. Frente al carácter tecnocrático que históricamente ha tenido la construcción de infraestructuras, en la medida en que constituyen aún la mayor inversión pública, se plantean la posibilidad de reivindicar su rol urbano, y no sólo urbanizador.

Esta vocación por entender las lógicas de las infraestructuras pero, también, por fijar criterios y estrategias de intervención se hace evidente en el trabajo de Rosina Vinyes. ${ }^{12}$ Su planteo del problema surge de las condiciones naturales de la ciudad contemporánea occidental, densa y compacta, y de las limitaciones del espacio urbano que hacen que el aprovechamiento del

\footnotetext{
${ }^{11}$ El Sistema Crítico es el ámbito físico y perceptivo de contacto entre la lógica técnica de implantación de la infraestructura de la movilidad y la matriz biofísica territorial.

${ }_{12}$ Sota Barcelona. La rellevància del subsòl en una ciutat contemporània. Investigación en curso de Rosina Vinyes. Director: Ángel Martín
} 
ID_INVESTIGACIONES

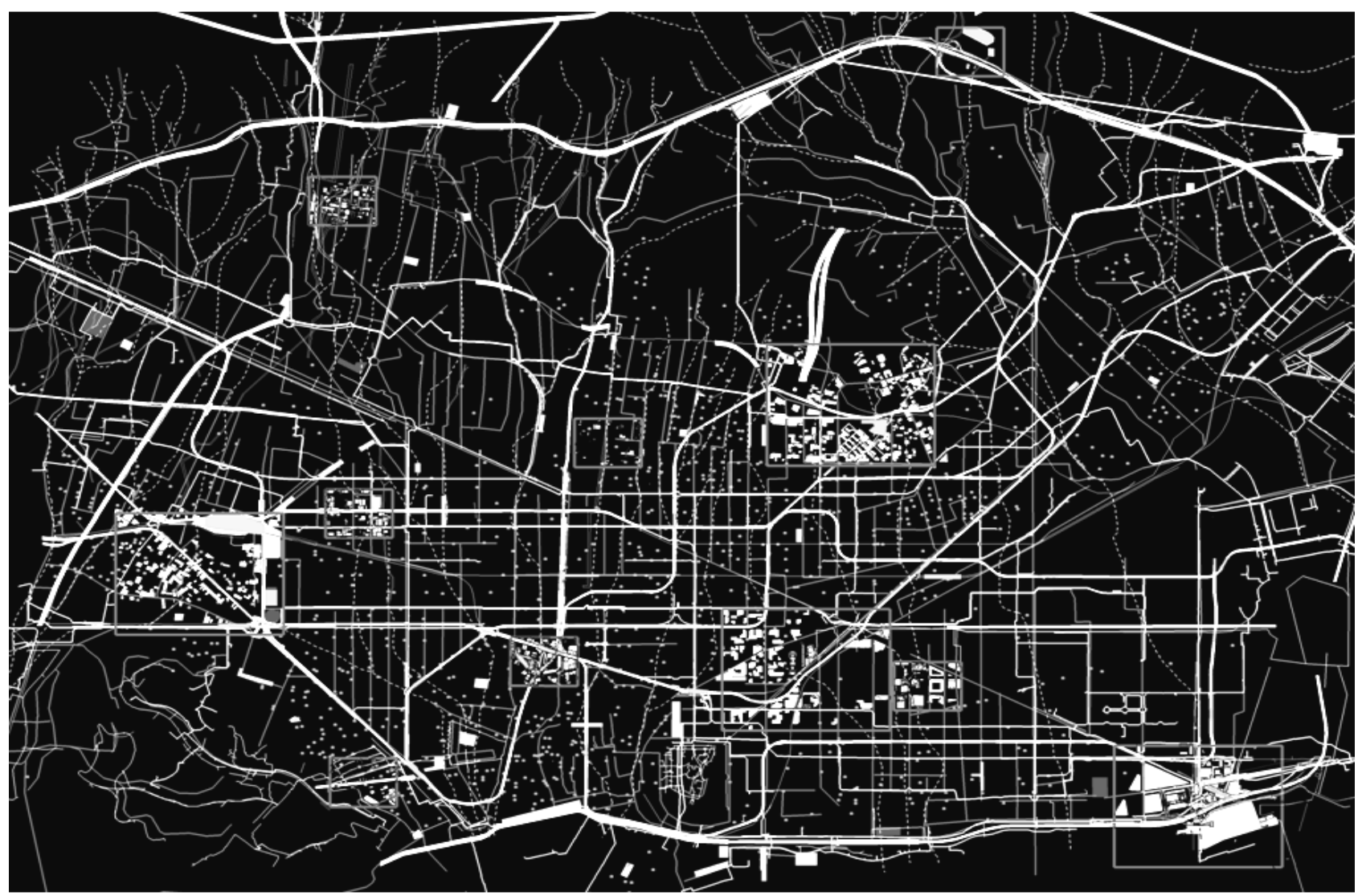

Barcelona, subsuelo

Ámbitos de estudio.

Fuente: Rosina Vinyes 
subsuelo sea cada vez mayor. La utilización del subsuelo no constituye una estrategia novedosa, ha estado vinculada secularmente al desarrollo de la ciudad. No obstante, debido a la dependencia creciente entre las ciudades y su subsuelo, resulta necesario estudiar las oportunidades potenciales. La reivindicación del papel protagonista del subsuelo en el urbanismo contemporáneo consolida la base para un mejor gobierno urbano.

Una segunda investigación parte del reconocimiento de los nuevos códigos de relación cívica que se dan en la metrópolis contemporánea. El problema que plantea Joan Moreno ${ }^{13}$ radica en la falta de elementos de referencia simbólica que se da en los espacios de la movilidad, en el territorio de las redes. Su hipótesis sostiene que las estrategias de intervención sobre el "sistema crítico", el espacio de mayor afluencia y continuidad de la región metropolitana, deben incidir en su naturaleza dual, es decir, en su condición segregada y potencial para estructurar el espacio libre de margen y establecer un lenguaje de escala apropiada. Sus argumentaciones se basan en la experiencia del caso holandés, específicamente de las infraestructuras que vertebran la Randstad y, a nivel local, en el caso del Corredor del Llobregat en su condición de "nueva Rambla Metropolitana".

Un desafío para estas investigaciones y futuros desarrollos radica en la consideración no tan sólo el diseño del objeto, sino también en los argumentos objetivos de posibles modelos y los instrumentos que los construyen y legitiman.

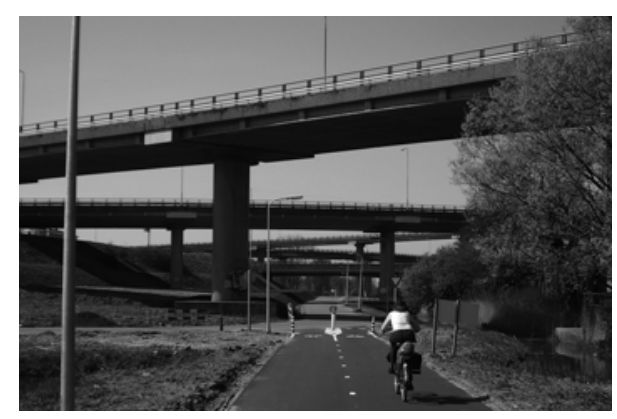

Randstad.

Fuente: Joan Moreno 


\section{EL ESTUDIO DE LAS CIUDADES LATINOAMERICANAS}

En sintonía con la tesis de Verena Andreatta, ${ }^{14}$ el desarrollo de un importante número de investigaciones asociadas al desarrollo de "la ciudad latinoamericana" se presenta como la oportunidad de consolidar un punto de vista complementario a las líneas canónicas desarrolladas a nivel local, especialmente, aquellas que parten de una metodología de cariz historicista. Algunas de las investigaciones desarrolladas en el DUOT siguen la línea que marca la hipótesis de Andreatta $(2007,2009)$ sobre la repercusión de la "circulación de ideas" como explicación de la formación de las ciudades latinoamericanas. Sin embargo, otro enfoque relevante radica en el análisis morfológico de estas ciudades consecuente con un tipo de aproximación arraigado desde hace más de treinta años en la Escuela de Barcelona.

Estos dos enfoques, entre otros tantos, definen un nuevo rasgo de la ciudad latinoamericana a la luz de una visión "desde fuera", que reivindica una tradición propia así como sus personajes protagonistas. Dentro de estos dos enfoques cabria mencionar dos investigaciones recientes. Por un lado, el trabajo de Mariana Debat ${ }^{15}$ parte de un nuevo enfoque frente a los estudios más tradicionales en el campo del urbanismo que sostienen que las ciudades latinoamericanas se construyen dominantemente a partir de una transferencia directa de modelos de otras latitudes, sin tener en cuenta el contexto local o las pre-existencias. La autora reivindica el papel de las decisiones voluntarias en contextos histórico-sociales diversos, citando a Bacon y Rykwert, y confirma la existencia de una amplia cultura urbana en la formación de algunas ciudades latinoamericanas, como muestra con el análisis de diversos planes y proyectos.

\footnotetext{
${ }^{13}$ El Sistema Crític. L'espai residual de la mobilitat contemporània com a agent estructurant de la realita metropolitana". Investigación en curso de Joan Moreno. Director: Miquel Martí

14 ANDREATTA, Verena (2007). Ciudades cuadradas, paraísos circulares. Planes de ordenación y orígenes de la urbanística en Río de Janeiro. Director de tesis: Joaquín Sabaté, Codirector de tesis: Manuel Herce, UPC, DUOT

${ }^{15}$ Ideas, reflexión e identidad. Seis episodios superpuestos en la construcción de Córdoba y Rosario.
} Investigación en curso de Mariana Debat. Director: Joaquín Sabaté

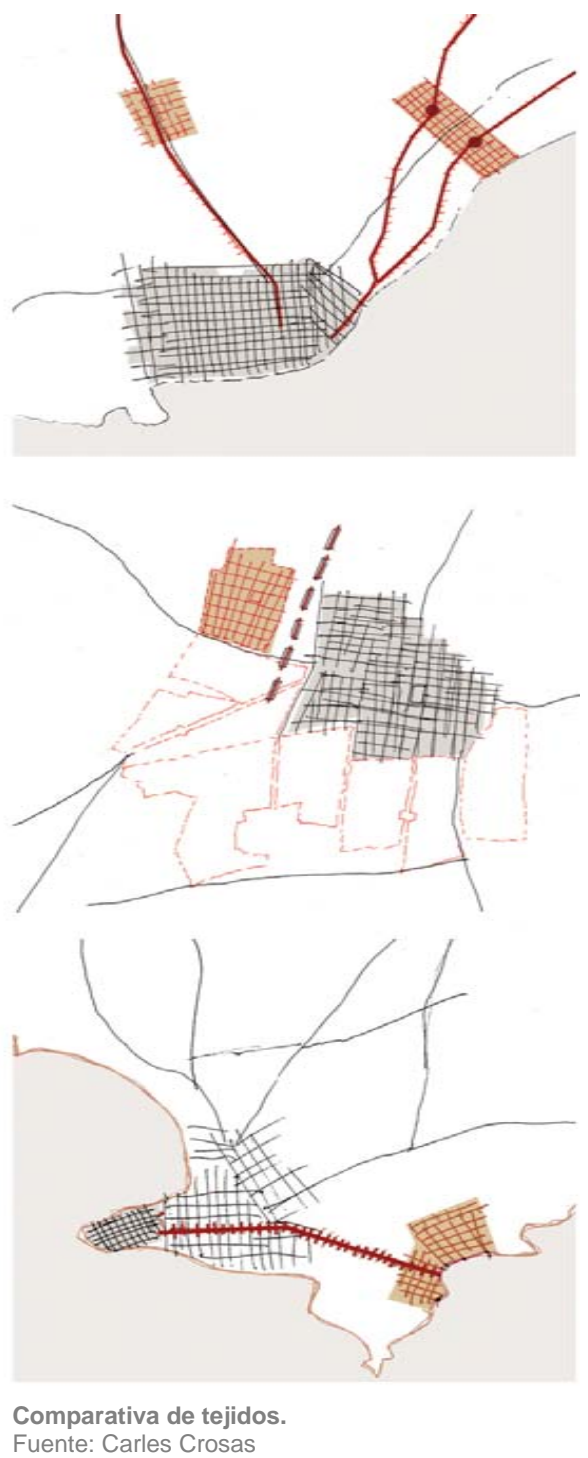


Su trabajo se basa en el estudio de los casos de Córdoba y Rosario, y adquiere un valor reivindicativo, al tratarse de ciudades de segundo orden.

La investigación de Carles Crosas $^{16}$ sobre el Vedado de La Habana recorre, quizás, el camino inverso a la antes mencionada. Parte del interés urbanístico de la propuesta de este "ensanche jardín", de la cultura híbrida y sin referentes directos (se encontraría entre las antiguas ciudades de fundación), de los contemporáneos ensanches españoles y de la creciente cultura del suburbio anglosajón, para determinar sus aportaciones morfológicas en la definición de un modelo. Asimismo, analiza la tipología de ciudad, en la que el deseo de la naturaleza (el verde) se superpone al orden reticular como expresión clásica de lo urbano y donde el ejemplo del Vedado se contextualiza dentro de un grupo de experiencias análogas en algunas capitales latinoamericanas (Buenos Aires, México DF, Montevideo y São Paulo).

A través de las cuatro líneas analizadas podríamos esbozar algunas reflexiones sobre la dirección de estas investigaciones y su especificidad.

Los instrumentos de planificación en Cataluña, ante una crisis del urbanismo occidental, se enfrentan a la recuperación de la legitimidad social de la disciplina y a la renovación de su cuerpo instrumental. A partir de su protagonismo actual las investigaciones se mueven entre la "optimización" y la "receptividad" de sus políticas, y podemos deducir que uno de los desafíos actuales de dichos instrumentos radica en superar dicha disyuntiva.

En segundo lugar, frente a la incertidumbre de si el "nuevo turismo" es capaz de poner en valor el paisaje, las investigaciones sobre el desarrollo turístico desde una perspectiva territorial, nos permiten reflexionar sobre su principal recurso, el paisaje, y las distintas formas de intervenir en él. Los paisajes del turismo abren una nueva perspectiva para la comprensión de un fenómeno, pero también para la definición de nuevos conceptos urbanísticos. El rasgo

${ }^{16}$ CROSAS, Carles (2009). Variaciones sobre la reularidad. El proyecto de El Vedado en la formación de La Habana Metropolitana. Dir: Xabier Eizaguirre, UPC, DUOT 
más innovador de este grupo radica en las categorías de análisis e intervención del turismo litoral, lo que de por sí, suponen una clara aportación disciplinar.

La tercera línea de investigación se opone a la idea ortodoxa de estructura urbana propia de la ciudad tradicional para evidenciar las dificultades del proyecto de los sistemas e infraestructuras como espacio público. Las tesis en curso reivindican su rol urbano, y no sólo de la urbanización. La manifestación autónoma de las infraestructuras, el principal capital fijo de la ciudad y el territorio, demuestra el interés y la pertinencia del proyecto del espacio de lo público, capaz de establecer un lenguaje consecuente con su escala.

Finalmente, la cuarta línea se basa en dos posibles enfoques, entre otros tantos, que definen una nueva dimensión urbana de la ciudad latinoamericana. La repercusión de la "circulación de ideas" y el análisis morfológico de las ciudades contribuyen a la reivindicación de una identidad latinoamericana, refutando la idea de que sus razones históricas y formativas eran deudoras de los modelos y visiones extranjeras, especialmente europeas.

Creo que el conjunto de estas investigaciones definen cuatro líneas relevantes que actualmente se desarrollan en el DUOT, evidenciando no sólo una aportación importante a los estudios urbanos, sino la posibilidad de plantear una nueva especificidad, híbrida, en las líneas de investigación en urbanismo. 


\section{BIBLIOGRAFÍA}

ASCHER, François (2007) [2004]. Los nuevos principios del urbanismo, Madrid, Alianza

BARBA, Rosa (1992). Peces mínimes i turisme. Quaderns d'arquitectura i Urbanisme. $\mathrm{n}^{\circ}$. 194. p 44-47. Barcelona

ANDREATTA, Verena (2007). Ciudades cuadradas, paraísos circulares. Planes de ordenación y orígenes de la urbanística en Río de Janeiro. Director de tesis: Joaquín Sabaté, Codirector de tesis: Manuel Herce, UPC, DUOT

CARACCIOLO, Román; DEBAT, Mariana; SABATÉ, Joaquín (ed.) (2008). I Seminario de investigación en urbanismo: actas. DUOT, UPC

CARACCIOLO, Román; DEBAT, Mariana; HORRACH, Biel; SABATÉ, Joaquín (ed.) (2008). II Seminario de investigación en urbanismo: actas. DUOT, UPC

CARACCIOLO, Román; DEBAT, Mariana; ELINBAUM, Pablo; HORRACH, Biel; MORENO, Joan; SABATÉ, Joaquín; VAN SLUYS, Christine (ed.) (2010). III Seminario de investigación en urbanismo: actas. DUOT, UPC

BUSQUETS, Joan; DOMINGO, Miquel; EIZAGUIRRE, Xavier; et al. (2003) Les formes urbanes del litoral català./ Ed. Diputació de Barcelona. Barcelona

FONT, Antonio. (ed.) (2007). La explosión de la ciudad. Transformaciones territoriales en las regiones urbanas de la Europa Meridional, Ministerio de Vivienda, Gobierno de España

MAGRINYÀ, Francesc (2010). Planificar l'Àrea Metropolitana des d'una lectura cerdaniana: urbanisme de xarxes i ecologia urbana. En: L'Agenda Cerdà: construint la Barcelona metropolitana. Joan Fuster (ed.); coordinació editorial. Ajuntament de Barcelona y Lunwerg, cop. Barcelona

MARTI, Miquel (2004). A la recerca de les civitas contemporània. Cap a una cultura urbana de l'espai públic: L'experiència de Barcelona (1979-2003). Tesis doctoral. Director: Joaquín Sabaté. UPC, DUOT

SABATĖ, Joaquín (1994). Tenerife. PIOT avance: plan insular de ordenación del territorio - Santa Cruz de Tenerife: El Cabildo,

VECSLIR, Lorena (2005). Paisajes de la nueva centralidad. Infraestructuras arteriales y polarización del crecimiento en la Región Metropolitana de Barcelona. Font, A. (director). Tesis doctoral, UPC, DUOT 